\title{
Co-dimension two bifurcations analysis of a delayed tumor model with Allee effect
}

\section{Qinrui Dai ${ }^{*}$ (1)}

"Correspondence: dqrhit@qq.com

'School of information and

Engineering, Wuhan Huaxia

University of Technology, Wuhan,

China

\section{Springer}

\begin{abstract}
The mathematical model has become an important means to study tumor treatment and has developed with the discovery of medical phenomena. In this paper, we establish a delayed tumor model, in which the Allee effect is considered. Different from the previous similar tumor models, this model is mainly studied from the point of view of stability and co-dimension two bifurcations, and some nontrivial phenomena and conclusions are obtained. By calculation, there are at most two positive equilibria in the system, and their stability is investigated. Based on these, we find that the system undergoes Bautin bifurcation, zero-Hopf bifurcation, and Hopf-Hopf bifurcation with time delay and tumor growth rate as bifurcation parameters. The interesting thing is that there is a Zero-Hopf bifurcation, which is not common in tumor models, making abundant dynamic phenomena appear in the system. By using the bifurcation theory of functional differential equations, we calculate the normal form of these Co-dimension two bifurcations. Finally, with the aid of MATLAB package DDE-BIFTOOL, some numerical simulations have been performed to support our theoretical results. In particular, we obtain the bifurcation diagram of the system in the two parameter plane and divide its regions according to the bifurcation curves. Meanwhile, the phenomena of multistability and periodic coexistence of some regions can be also demonstrated. Combined with the simulation results, we can know that when the tumor growth rate and the delay of immune cell apoptosis are small, the tumor may tend to be stable, and vice versa.
\end{abstract}

Keywords: Allee effect; Bautin bifurcation; Zero-Hopf bifurcation; Hopf-Hopf bifurcation

\section{Introduction}

Cancer, also known as malignant tumor, grows fast and is very difficult to cure, which seriously affects people's life and health [1-4]. In recent years, with the vigorous development of biomathematics and interdisciplinary, it has become a new research trend to solve the related problems in biomedical field by establishing mathematical models. Therefore, many scholars have devoted themselves to the study of tumor models and made remarkable achievements, including tumor-immune and various types of tumor models, such as melanoma model, autoimmune disease model, hepatitis B model, and so on [5-7]. Most of them study the model from the perspective of stability and bifurcation, and consider the delay $[8,9]$ and other effects in the model. For example, the authors in reference [10] stud-

(c) The Author(s) 2021. This article is licensed under a Creative Commons Attribution 4.0 International License, which permits use, sharing, adaptation, distribution and reproduction in any medium or format, as long as you give appropriate credit to the original author(s) and the source, provide a link to the Creative Commons licence, and indicate if changes were made. The images or other third party material in this article are included in the article's Creative Commons licence, unless indicated otherwise in a credit line to the material. If material is not included in the article's Creative Commons licence and your intended use is not permitted by statutory regulation or exceeds the permitted use, you will need to obtain permission directly from the copyright holder. To view a copy of this licence, visit http://creativecommons.org/licenses/by/4.0/. 
ied the symmetric-breaking bifurcation properties of a tumor model with delay and free boundary. In [11], a three-dimensional ordinary differential equation model is established to explore the growth law of tumor cells, and its bifurcation and multistability are studied. These models have different definitions of tumor growth, most of which are exponential growth and logistic growth. However, many scholars have pointed out that the Allee effect exists not only in the macro population entities but also in the growth of tumors [12-17]. Therefore, considering the delay of apoptosis of immune cells and the Allee effect of tumor cell growth, the following functional differential equation model is established:

$$
\left\{\begin{array}{l}
\frac{d x}{d t}=\alpha \frac{y}{y+b}-d x(t-\tau), \\
\frac{d y}{d t}=r y\left(1-\frac{y}{k}\right)(y-m)-\beta x y,
\end{array}\right.
$$

where $x, y$ represent the number of human immune cells and tumor cells at time $t$ respectively. $\alpha, b$, and $d$ stand for the growth rate, semi-saturation constant, and apoptosis rate of immune cells respectively, and the delay of apoptosis is expressed by $\tau$. $r, \beta$ represent the growth rate and apoptosis rate of tumor cells respectively. The maximum carrying capacity of tumor cells in human body is defined as $k$, and $m$ is Allee threshold. In particular, inspired by [18-20], we use $\alpha \frac{y}{y+b}$ instead of the usual immune cells growth term $\alpha y$, whose growth is independent of tumor cells, and for the apoptosis of tumor cells, we follow the models in $[19,21]$.

Although many scholars have studied models similar to (1), most of them stop at codimension one bifurcations, and there is less research on co-dimension two bifurcations. Co-dimension two bifurcations are usually accompanied by abundant dynamic phenomena, especially when there are multiple codimension two bifurcations in a model. Therefore, this paper focuses on some interesting dynamic phenomena of model (1) from the perspective of high co-dimension bifurcations.

The rest of this paper is organized as follows. In Sect. 2, we analyze the existence and stability of positive equilibria of system (1). Hopf bifurcation and Bautin bifurcation are investigated in Sect. 3, and the key values required for Bautin bifurcation analysis are calculated. We study the existence and normal form of zero-Hopf bifurcation in Sect. 4. In Sect. 5, we analyze another co-dimension two bifurcation, namely Hopf-Hopf bifurcation. To support our theoretical analysis, the two-parameter bifurcation diagram is obtained in Sect. 6, and given the bifurcation diagram, we give some numerical simulations for different parameters. Finally, a brief conclusion and expectation section completes the paper.

\section{Existence and stability of positive equilibria}

It is obvious that system (1) has a zero equilibrium $E_{0}=(0,0)$ and no boundary equilibrium. Considering the positive equilibrium $E^{*}\left(x^{*}, y^{*}\right)$, one satisfies

$$
\left\{\begin{array}{l}
\alpha \frac{y^{*}}{y^{*}+b}-d x^{*}=0, \\
r y^{*}\left(1-\frac{y^{*}}{k}\right)\left(y^{*}-m\right)-\beta x^{*} y^{*}=0 .
\end{array}\right.
$$

After sorting out equation (2), we can get the following result:

$$
\left(y^{*}+b\right)\left(y^{*}-k\right)\left(y^{*}-m\right)+\frac{k \alpha \beta}{d r} y^{*}=0 .
$$


Let

$$
f\left(y^{*}\right)=\left(y^{*}+b\right)\left(y^{*}-k\right)\left(y^{*}-m\right)+\frac{k \alpha \beta}{d r} y^{*},
$$

and for convenience, we give the derivative of (3)

$$
f^{\prime}\left(y^{*}\right)=3\left(y^{*}\right)^{2}+2(b-k-m) y^{*}+k m+\frac{k \alpha \beta}{d r}-b(k+m) .
$$

The two zeros of function (4) are

$$
y_{1}^{*}=\frac{-2(b-k-m)-\sqrt{\Delta}}{6}, \quad y_{2}^{*}=\frac{-2(b-k-m)+\sqrt{\Delta}}{6},
$$

where

$$
\Delta=4(b-k-m)^{2}-12\left[k m+\frac{k \alpha \beta}{d r}-b(k+m)\right] .
$$

Using some basic knowledge of quadratic function, we have the following.

Theorem 2.1 For system (1).

(1) If $y_{1}^{*}>0, \Delta>0$, and $f\left(y_{2}^{*}\right)<0$ all hold, then function (3) has two different zeros, that is, system (1) has two different positive equilibria, denoted by $E_{1}\left(x_{1}, y_{1}\right)$ and $E_{2}\left(x_{2}, y_{2}\right)$.

(2) If $y_{1}^{*}>0, \Delta>0$, and $f\left(y_{2}^{*}\right)=0$ are all satisfied, then there is a unique zero for function (3), and the corresponding equilibrium of system (1) is denoted by $E_{3}\left(x_{3}, y_{3}\right)$.

Without loss of generality, this paper focuses on the stability of positive equilibrium $E_{2}$. The linearization of system (1) at this equilibrium is actually

$$
\left\{\begin{array}{l}
\frac{d x}{d t}=a_{12} y-d x(t-\tau), \\
\frac{d y}{d t}=a_{22} y-a_{21} x,
\end{array}\right.
$$

where

$$
\begin{aligned}
& a_{12}=\alpha \frac{b}{\left(y_{2}+b\right)^{2}}, \quad a_{21}=\beta y_{2}, \\
& a_{22}=r\left[\left(1-\frac{2 y_{2}}{k}\right)\left(y_{2}-m\right)+\left(y_{2}-\frac{y_{2}^{2}}{k}\right)\right]-\beta x_{2} .
\end{aligned}
$$

Thus, the characteristic equation of the system can be easily obtained as follows:

$$
\lambda^{2}-a_{22} \lambda+\left(d \lambda-a_{22} d\right) e^{-\lambda \tau}+a_{12} a_{21}=0 .
$$

When $\tau=0$, equation (6) becomes

$$
\lambda^{2}+\left(d-a_{22}\right) \lambda+a_{12} a_{21}-a_{22} d=0,
$$


and according to the Routh-Hurwitz criterion, all roots of the characteristic equation have negative real parts when $d>a_{22}$ and $a_{12} a_{21}>a_{22} d$ hold. That is to say, in this case, the positive equilibrium $E_{2}$ is locally asymptotically stable.

When $\tau>0$, suppose that $\lambda_{1,2}= \pm i \omega$ are the roots of equation (6), and take it into the equation

$$
-\omega^{2}-i a_{22} \omega+\left(i d \omega-a_{22} d\right)(\cos \omega \tau-i \sin \omega \tau)+a_{12} a_{21}=0 .
$$

Separating the real and imaginary parts of (7), we can get

$$
\left\{\begin{array}{l}
d \omega \sin \omega \tau-a_{22} d \cos \omega \tau=\omega^{2}-a_{12} a_{21}, \\
d \omega \cos \omega \tau+a_{22} d \sin \omega \tau=a_{22} \omega .
\end{array}\right.
$$

By squaring both sides of (8) and adding the two equations, the following result can be obtained:

$$
\omega^{4}+\left(a_{22}^{2}-2 a_{12} a_{21}-d^{2}\right) \omega^{2}+a_{11}^{2} a_{21}^{2}-a_{22}^{2} d^{2}=0 .
$$

Let $v=\omega^{2}$, and equation (9) is now

$$
v^{2}+\eta_{1} v+\eta_{2}=0
$$

and the corresponding two roots are expressed as

$$
v_{1}=\frac{-\eta_{1}+\sqrt{\eta_{1}-4 \eta_{2}}}{2}, \quad v_{2}=\frac{-\eta_{1}-\sqrt{\eta_{1}-4 \eta_{2}}}{2}
$$

It is easy to see that when $\eta_{1}-4 \eta_{2}>0$ and $-\frac{\eta_{1}}{2}>0$ hold, equation (10) has positive roots, and without loss of generality, suppose that $v_{1}$ and $v_{2}$ are positive roots. Accordingly, equation (9) has two positive solutions $\omega_{1}=\sqrt{v_{1}}$ and $\omega_{2}=\sqrt{v_{2}}$, and the corresponding critical value of time delay is

$$
\tau_{n}^{(j)}=\left\{\begin{array}{l}
\frac{1}{\omega_{n}}\left[\arccos \left\{\frac{a_{12} a_{21} a_{22}}{\left(a_{22}^{2}+w^{2}\right) d}\right\}+2 j \pi\right], \quad \cos \omega \tau>0, \\
\frac{1}{\omega_{n}}\left[\pi-\arccos \left\{\frac{a_{12} a_{21} a_{22}}{\left(a_{22}^{2}+w^{2}\right) d}\right\}+2 j \pi\right], \quad \cos \omega \tau<0,
\end{array}\right.
$$

where $n=1,2$ and $j=1,2,3, \ldots$ Defining $\tau_{0}=\min \left\{\tau_{n}^{(j)}\right\}$, the corresponding $\omega_{n}$ is $\omega_{0}$. Let $\lambda=\xi(\tau)+i \omega(\tau)$ be the root of equation (6), where $\xi$ and $\omega$ satisfy $\xi\left(\tau_{0}\right)=0$ and $\omega\left(\tau_{0}\right)=\omega_{0}$, respectively. Differentiating both sides of the characteristic equation (6) with respect to $\lambda$ gives

$$
2 \lambda \frac{d \lambda}{d \tau}-a_{22} \frac{d \lambda}{d \tau}+d e^{-\lambda \tau} \frac{d \lambda}{d \tau}-d \lambda e^{-\lambda \tau}\left[\frac{d \lambda}{d \tau} \tau+\lambda\right]+a_{22} d e^{-\lambda \tau}\left[\frac{d \lambda}{d \tau} \tau+\lambda\right]=0,
$$

and then we have

$$
\left[\frac{d \lambda}{d \tau}\right]^{-1}=\frac{\left(2 \lambda-a_{22}\right) e^{\lambda \tau}}{\lambda\left(d \lambda-a_{22} d\right)}+\frac{d}{\lambda\left(d \lambda-a_{22} d\right)}-\frac{\tau}{\lambda} .
$$


Obviously, the transversality condition $\operatorname{sign}\left[\frac{d \lambda}{d \tau}\right]_{\tau=\tau_{0}} \neq 0$ if and only if $a_{22}^{2}+2 \omega_{0}^{2}-d^{2}-$ $2 a_{12} a_{21} \neq 0$, where

$$
\begin{aligned}
\operatorname{sign}\left[\frac{d \lambda}{d \tau}\right]_{\tau=\tau_{0}} & =\operatorname{sign}\left[\operatorname{Re}\left(\frac{d \lambda}{d \tau}\right)\right]^{-1} \\
& =\operatorname{sign}\left\{\operatorname{Re}\left[\frac{\left(2 \lambda-a_{22}\right) e^{\lambda \tau}+d}{\lambda\left(d \lambda-a_{22} d\right)}\right]\right\} \\
& =\operatorname{sign}\left\{\operatorname{Re}\left[\frac{\left(2 i \omega_{0}-a_{22}\right)\left(\cos i \omega_{0}+i \sin i \omega_{0}\right)+d}{i \omega_{0}\left(i d \omega_{0}-a_{22} d\right)}\right]\right\} \\
& =\operatorname{sign}\left[\frac{a_{22}^{2}+2 \omega_{0}^{2}-d^{2}-2 a_{12} a_{21}}{d^{2}\left(\omega_{0}^{2}+a_{22}^{2}\right)}\right] .
\end{aligned}
$$

Theorem 2.2 The positive equilibrium $E_{2}$ is locally asymptotically stable(unstable) when $\tau \in\left(0, \tau_{0}\right)\left(\tau>\tau_{0}\right)$, and system (1) undergoes Hopf bifurcation at positive equilibrium $E_{2}$ when $\tau=\tau_{0}$ and $a_{22}^{2}+2 \omega_{0}^{2}-d^{2}-2 a_{12} a_{21} \neq 0$.

\section{Bautin bifurcation}

When the first Lyapunov coefficient is equal to 0 , the Hopf bifurcation may degenerate and Bautin bifurcation occurs. In this section, Bautin bifurcation is investigated at positive equilibrium $E_{2}$ with $\tau$ and $r$ as bifurcation parameters. Next, according to the research in [22-24], the properties of Hopf bifurcation and Bautin bifurcation are studied. Let $\left(\tau_{0}, r_{0}\right)$ be the Bautin bifurcation point for bifurcation analysis. After scaling $t \rightarrow \frac{t}{\tau}$ and introducing $\mu=\tau-\tau_{0}, \varsigma=r-r_{0}, u_{1}=x(t)-x_{2}, u_{2}(t)=y(t)-y_{2}$, system (1) can be written as

$$
\left\{\begin{aligned}
\dot{u}_{1}(t)= & \left(\tau_{0}+\mu\right)\left\{a_{12} u_{2}(t)-d u_{1}(t-1)\right. \\
& \left.-a_{13} u_{2}^{2}(t)+a_{14} u_{2}^{3}(t)+\text { h.o.t }\right\} \\
\dot{u}_{2}(t)= & \left(\tau_{0}+\mu\right)\left\{a_{21} u_{1}(t)+a_{22}^{*} u_{2}(t)+a_{23} u_{2}^{2}(t)\right. \\
& \left.-\beta u_{1}(t) u_{2}(t)-a_{24} u_{2}^{3}(t)\right\}
\end{aligned}\right.
$$

where

$$
\begin{aligned}
& a_{13}=\frac{\alpha b}{\left(y_{2}+b\right)^{3}}, \quad a_{14}=\frac{\alpha b}{\left(y_{2}+b\right)^{4}}, \\
& a_{22}^{*}=\left(r_{0}+\varsigma\right)\left[\left(1-\frac{2 y_{2}}{k}\right)\left(y_{2}-m\right)+\left(y_{2}-\frac{y_{2}^{2}}{k}\right)\right]-\beta x_{2}, \\
& a_{23}=\left(r_{0}+\varsigma\right)\left[\left(1+\frac{m}{k}\right)-\frac{3}{k} y_{2}\right], \quad a_{24}=\frac{r_{0}+\varsigma}{k} .
\end{aligned}
$$

The above system (11) is transformed into a functional differential equation on the phase space $C=C\left([-1,0], \mathbb{R}^{2}\right)$

$$
\dot{u}(t)=L(\mu, \varsigma) u_{t}+F\left(\mu, \varsigma, u_{t}\right)
$$

where $u(t)=\left(u_{1}(t), u_{2}(t)\right)^{T} \in \mathbb{R}^{2}, L: C \rightarrow \mathbb{R}^{2}, u_{t}=u(t+\theta), \theta \in[-1,0]$, and $F: \mathbb{R}^{2} \times C \rightarrow \mathbb{R}^{2}$. For $\varphi(\theta)=\left(\varphi_{1}(\theta), \varphi_{2}(\theta)\right) \in C$, we have

$$
L(\mu, \varsigma) \varphi=B_{1} \varphi(0)+B_{2} \varphi(-1) .
$$


where

$$
B_{1}=\tau_{0}\left(\begin{array}{cc}
0 & a_{12} \\
a_{21} & a_{22}^{*}
\end{array}\right), \quad B_{2}=\tau_{0}\left(\begin{array}{cc}
-d & 0 \\
0 & 0
\end{array}\right)
$$

and

$$
\begin{aligned}
F(\mu, \zeta, \varphi)= & \left(\begin{array}{c}
\mu\left(a_{12} \varphi_{2}(0)-d \varphi_{1}(0)\right) \\
\mu\left(a_{21} \varphi_{1}(0)-a_{22}^{*} \varphi_{2}(0)\right)+\tau_{0} \zeta a_{22}^{*} \varphi_{2}(0)
\end{array}\right) \\
& +\left(\begin{array}{c}
\left(\tau_{0}+\mu\right)\left\{-a_{13} \varphi_{2}^{2}(0)+a_{14} \varphi_{2}^{3}(0)+\text { h.o.t }\right\} \\
\left(\tau_{0}+\mu\right)\left\{a_{23} \varphi_{2}^{2}(0)-\beta \varphi_{1}(0) \varphi_{2}(0)+a_{24} \varphi_{2}^{3}(0)\right\}
\end{array}\right) .
\end{aligned}
$$

By the Riesz representation theorem, there exists bounded variation functions $\eta(\theta, \mu, \varsigma)$ in $\theta \in[-1,0]$ such that

$$
L(\mu, \varsigma) \varphi=\int_{-1}^{0} d \eta(\theta, \mu, \varsigma) \varphi(\theta), \quad \text { for } \varphi \in C[-1,0] .
$$

In fact, $\eta(\theta, \mu, \varsigma)$ can be

$$
d \eta(\theta, \mu, \varsigma)=B_{1} \delta(\theta)+B_{2} \delta(\theta+1),
$$

where $\delta$ is the Dirac delta function. Now define operator

$$
A(\mu, \varsigma) \varphi= \begin{cases}\frac{d \varphi(\theta)}{d \theta}, & \text { for } \theta \in[-1,0), \\ \int_{-1}^{0} d \eta(\theta, \mu, \varsigma) \varphi(\theta), & \text { for } \theta=0\end{cases}
$$

and

$$
R(\mu, \varsigma) \varphi= \begin{cases}0, & \text { for } \theta \in[-1,0), \\ F(\mu, \varsigma, \theta), & \text { for } \theta=0 .\end{cases}
$$

System (11) is equivalent to the following abstract ordinary differential equation:

$$
\dot{u}_{t}=A(\mu) u_{t}+R(\mu) u_{t}
$$

where $u_{t}=u(t+\theta), \theta \in[-1,0]$. The adjoint operator $A^{*}$ of $A$ is

$$
A^{*}(\mu, \varsigma) \psi= \begin{cases}-\frac{d \psi(s)}{d s}, & \text { for } s \in(0,1] \\ \int_{-1}^{0} \psi(-s) d \eta(s, \mu, \varsigma), & \text { for } s=0\end{cases}
$$

For $\varphi \in C[-1,0]$ and $\psi \in C[0,1]$, define the bilinear form

$$
\langle\psi(s), \varphi(\theta)\rangle=\bar{\psi}(0) \varphi(0)-\int_{-1}^{0} \int_{0}^{\theta} \bar{\psi}(\xi-\theta) d \eta(\theta) \varphi(\xi) d \xi
$$


where $\eta(\theta)=\eta(\theta, 0,0)$. It is easy to show $\langle\psi, A \varphi\rangle=\left\langle A^{*} \psi, \varphi\right\rangle$. Let $q(\theta)$ be the eigenvector corresponding to eigenvalue $i \omega_{0}$ of $A(0,0)$ and $q^{*}(s)$ be the eigenvector corresponding to eigenvalue $-i \omega_{0}$ of $A^{*}$, namely

$$
A(0,0) q(\theta)=i \omega_{0} q(\theta), \quad A^{*} q^{*}(s)=-i \omega_{0} q^{*}(s) .
$$

By simple calculation, we have

$$
q(\theta)=\left(1, q_{1}\right)^{T} e^{i \omega_{0} \theta}, \quad q^{*}(s)=D\left(1, q_{1}^{*}\right)^{T} e^{i \omega_{0} s},
$$

where

$$
\begin{aligned}
& q_{1}=\frac{i \omega_{0}-d e^{i \omega_{0} \tau_{0}}}{a_{12}}, \quad q_{1}^{*}=\frac{-i \omega_{0}-d e^{-i \omega_{0} \tau_{0}}}{a_{12}}, \\
& \bar{D}=\frac{1}{1+\bar{q}_{1}^{*} q_{1}-d \tau_{0} e^{-i \omega_{0} \tau_{0}}} .
\end{aligned}
$$

Defining $z(t)=\left\langle q^{*}, u_{t}\right\rangle$ and $W^{(\mu, 5)}(t, \theta)=u_{t}(\theta)-2 \operatorname{Re}[z(t) q(\theta)]$, on the center manifold $C_{0}$, we have

$$
W^{(\mu, 5)}(t, \theta)=W^{(\mu, \zeta)}(z(t), \bar{z}(t), \theta)
$$

and

$$
\begin{aligned}
W^{(\mu, \zeta)}(z(t), \bar{z}(t), \theta)= & W_{20}^{(\mu, \varsigma)}(\theta) \frac{z^{2}}{2}+W_{11}^{(\mu, \varsigma)}(\theta) z \bar{z} \\
& +W_{02}^{(\mu, \varsigma)}(\theta) \frac{\bar{z}^{2}}{2}+W_{30}^{(\mu, \varsigma)}(\theta) \frac{z^{3}}{6}+\cdots
\end{aligned}
$$

For convenience, rewriting $W_{i j}^{(\mu, \varsigma)}$ as $W_{i j}$, this is $W_{i j}^{(\mu, \varsigma)}=\left(W_{i j}^{(1)}, W_{i j}^{(2)}\right), i+j \geq 2$, we have

$$
\begin{aligned}
\dot{z}(t) & =\left\langle q^{*}, \dot{u}_{t}\right\rangle=\left\langle q^{*}, A u_{t}+R u_{t}\right\rangle \\
& =\left\langle A^{*} q^{*}, u_{t}\right\rangle+\left\langle q^{*}, R u_{t}\right\rangle \\
& =i \omega_{0} z+\bar{q}^{*}(0) F\left(0, W^{(\mu, \varsigma)}(t, \theta)+2 \operatorname{Re}[z(t) q(0)]\right) \\
& =i \omega_{0} z+g(z, \bar{z}),
\end{aligned}
$$

where

$$
g(z, \bar{z})=\sum_{i+j \geq 2} g_{i j} \frac{1}{i ! j !} z^{i} \bar{z}^{j}
$$

Moreover, one obtains

$$
\begin{aligned}
g(z, \bar{z}) & =\bar{q}^{*}(0) F(z, \bar{z}) \\
& =\left(\tau_{0}+\mu\right) D\left(1 \bar{q}_{1}^{*}\right)\left(\begin{array}{c}
-a_{13} u_{2}^{2}(0)+a_{14} u_{2}^{2}(0)+\text { h.o.t } \\
a_{23} u_{2}^{2}(0)-\beta u_{1}(0) u_{2}(0)-a_{24} u_{2}^{2}(0)
\end{array}\right) .
\end{aligned}
$$


The expression of $u_{t}(\theta)=\left(u_{1 t}(\theta), u_{2 t}(\theta)\right)^{T}$ is easy to get

$$
\left\{\begin{aligned}
u_{1}(0)= & z+\bar{z}+W_{20}^{(1)}(0) \frac{z^{2}}{2} \\
& +W_{11}^{(1)}(0) z \bar{z}+W_{02}^{(1)}(0) \frac{\bar{z}^{2}}{2}+\cdots \\
u_{2}(0)= & q_{1} z+\bar{q}_{1} \bar{z}+W_{20}^{(2)}(0) \frac{z^{2}}{2} \\
& +W_{11}^{(2)}(0) z \bar{z}+W_{02}^{(2)}(0) \frac{\bar{z}^{2}}{2}+\cdots
\end{aligned}\right.
$$

Substituting (14) into (13), then comparing the coefficient with (12), we can get

$$
\begin{aligned}
& g_{21}=2\left(M_{1} G_{210}-M_{2} G_{211}\right), \quad g_{02}=2\left(M_{1} \bar{q}_{1}^{2}-M_{2} \bar{q}_{1}\right) \\
& g_{20}=2\left(M_{1} q_{1}^{2}-M_{2} q_{1}\right), \\
& g_{11}=M_{1} q_{1} \bar{q}_{1}-\beta \bar{q}_{1}^{*} D\left(q_{1}+\bar{q}_{1}\right)\left(\tau_{0}+\mu\right), \quad g_{12}=2\left(M_{1} G_{120}-M_{2} G_{121}\right), \\
& g_{40}=12\left(2 M_{1} G_{400}-M_{2} G_{401}\right), \quad g_{31}=3\left(2 M_{1} G_{310}-M_{2} G_{311}\right) \\
& g_{13}=3\left(2 M_{1} G_{130}-M_{2} G_{131}\right), \quad g_{32}=6\left(2 M_{1} G_{320}-M_{2} G_{321}\right) \\
& g_{22}=4\left(M_{1} G_{220}-M_{2} G_{221}\right), \\
& g_{03}=6 M_{1} \bar{q}_{1} W_{02}^{(2)}-6 M_{2}\left(\frac{\bar{q}_{1} W_{02}^{(1)}(0)}{2}+W_{02}^{(2)}(0)\right) \\
& g_{30}=6 M_{1} q_{1} W_{20}^{(2)}(0)-6 M_{2}\left(\frac{q_{1} W_{20}^{(1)}(0)}{2}+W_{20}^{(2)}(0)\right)
\end{aligned}
$$

where

$$
\begin{aligned}
& M_{2}=\beta \bar{q}_{1}^{*} D\left(\tau_{0}+\mu\right), \quad M_{1}=\left(\tau_{0}+\mu\right)\left[D\left(a_{14}-a_{13}\right)+D \bar{q}_{1}^{*}\left(a_{23}-a_{24}\right)\right] \\
& G_{210}=\bar{q}_{1} W_{20}^{(2)}(0)+2 q_{1} W_{11}^{(2)}(0), \quad G_{120}=q_{1} W_{02}^{(2)}(0)+2 \bar{q}_{1} W_{11}^{(2)}(0), \\
& G_{400}=\frac{q_{1} W_{30}^{(2)}+\frac{\left(W_{20}^{(2)}\right)^{2}}{4}, \quad G_{310}=q_{1} W_{21}^{(2)}(0)+\frac{\bar{q}_{1} W_{30}^{(2)}(0)}{3},}{G_{130}=} \bar{q}_{1} W_{12}^{(2)}(0)+\frac{q_{1} W_{03}^{(2)}(0)}{3}, \quad G_{221}=W_{11}^{(1)}(0) W_{11}^{(2)}(0)+\frac{H_{2}}{2}, \\
& G_{311}=\frac{\bar{q}_{1} W_{30}^{(1)}(0)+W_{30}^{(2)}(0)}{3}+q_{1} W_{21}^{(1)}(0)+W_{21}^{(2)}(0), \\
& G_{401}=\frac{q_{1} W_{30}^{(1)}(0)+W_{30}^{(2)}(0)}{3}+\frac{W_{20}^{(1)}(0) W_{20}^{(2)}(0)}{2}, \\
& G_{121}=\frac{\bar{q}_{1} W_{02}^{(1)}(0)+W_{02}^{(2)}(0)}{2}+\bar{q}_{1} W_{11}^{(1)}(0)+W_{11}^{(2)}(0), \\
& G_{211}=\frac{\bar{q}_{1} W_{20}^{(1)}(0)+W_{20}^{(2)}(0)}{2}+q_{1} W_{11}^{(1)}(0)+W_{11}^{(2)}(0), \\
& G_{131}=\frac{q_{1} W_{03}^{(1)}(0)+W_{03}^{(2)}(0)}{3}+\bar{q}_{1} W_{12}^{(1)}(0)+W_{12}^{(2)}(0), \\
& G_{320}= \\
& W_{21}^{(2)}(0) W_{11}^{(2)}(0)+\frac{2 \bar{q}_{1}}{} W_{31}^{(2)}(0)+W_{30}^{(2)}(0) W_{02}^{(2)}(0) \\
& 6
\end{aligned}
$$




$$
\begin{aligned}
& G_{321}=W_{21}^{(1)}(0) W_{11}^{(2)}(0)+W_{21}^{(2)}(0) W_{11}^{(1)}(0)+\frac{\bar{q}_{1} W_{31}^{(1)}(0)+W_{31}^{(2)}(0)}{3}+\frac{H_{1}}{6}, \\
& G_{220}=\bar{q}_{1} W_{21}^{(2)}(0)+q_{1} W_{12}^{(2)}(0)+\left(W_{11}^{(2)}(0)\right)^{2}+\frac{W_{20}^{(2)}(0) W_{02}^{(2)}(0)}{2},
\end{aligned}
$$

and

$$
\begin{aligned}
H_{1}= & W_{30}^{(1)}(0) W_{02}^{(2)}(0)+W_{30}^{(2)}(0) W_{02}^{(1)}(0) \\
H_{2}= & \bar{q}_{1} W_{21}^{(1)}(0)+q_{1} W_{12}^{(1)}(0)+W_{21}^{(2)}(0)+W_{12}^{(2)}(0) \\
& +W_{20}^{(1)}(0) W_{02}^{(2)}(0)+W_{02}^{(1)}(0) W_{20}^{(2)}(0)
\end{aligned}
$$

Furthermore, the calculation results show that

$$
\mu_{2}=-\frac{\operatorname{Re}\left[C_{1}(0)\right]}{\operatorname{Re}\left[\lambda^{\prime}\left(\tau_{0}\right)\right]}, \quad \beta_{2}=2 \operatorname{Re} C_{1}(0),
$$

where

$$
C_{1}(0)=\frac{i}{2 \omega_{0}}\left(g_{20} g_{11}-2\left|g_{11}\right|^{2}-\frac{1}{3}\left|g_{02}\right|^{2}\right)+\frac{g_{21}}{2} .
$$

The sign of $\mu_{2}$ reveals the direction of the Hopf bifurcation and $\beta_{2}$ the stability of periodic solutions, so we have the following.

Theorem 3.1 If $\mu_{2}>0\left(\mu_{2}<0\right)$, then the Hopf bifurcation is supercritical(subcritical) and the bifurcating periodic solutions on the center manifold are stable(unstable) if $\beta_{2}<0$ $\left(\beta_{2}>0\right)$.

Next, to illustrate the existence of Bautin bifurcation, we need to calculate the first Lyapunov coefficient and the second Lyapunov coefficient as follows:

$$
l_{1}(\mu, \varsigma)=\frac{1}{2 \omega_{0}}\left[\operatorname{Re} g_{21}-\frac{1}{\omega_{0}} \operatorname{Im}\left(g_{20} g_{11}\right)\right]
$$

and

$$
\begin{aligned}
12 l_{2}(0,0)= & \frac{1}{\omega_{0}} \operatorname{Re} g_{32}+\frac{1}{\omega_{0}^{2}} \operatorname{Im}\left[g_{20} \bar{g}_{31}-g_{11}\left(4 g_{31}+3 \bar{g}_{22}\right)-\frac{1}{3} g_{02}\left(g_{40}+\bar{g}_{13}\right)-g_{30} g_{12}\right] \\
& +\frac{1}{\omega_{0}^{3}} \operatorname{Re}\left[g_{20} \bar{g}_{11}\left(3 g_{12}-\bar{g}_{30}\right)+g_{20} g_{02}\left(\bar{g}_{12}-\frac{1}{3} g_{30}\right)\right. \\
& \left.+\frac{1}{3} g_{20} \bar{g}_{02} g_{30}+g_{11} \bar{g}_{02}\left(\frac{5}{3} \bar{g}_{30}+3 g_{12}\right)\right] \\
& +\frac{1}{\omega_{0}^{3}} \operatorname{Re}\left[\frac{1}{3} g_{11} g_{02} \bar{g}_{03}-4 g_{2}^{11} g_{30}\right]+\frac{3}{\omega_{0}^{3}} \operatorname{Im}\left(g_{20} g_{11}\right) \operatorname{Im} g_{21} \\
& +\frac{1}{\omega_{0}^{4}} \operatorname{Im}\left[g_{11} \bar{g}_{02}\left(\bar{g}_{20}^{2}-3 \bar{g}_{20} g_{11}-4 g_{11}^{2}\right]\right. \\
& +\frac{1}{\omega_{0}^{4}} \operatorname{Im}\left(g_{20} g_{11}\right)\left[3 \operatorname{Re}\left(g_{20} g_{11}\right)-2\left|g_{02}\right|^{2}\right] .
\end{aligned}
$$


For the transversality condition, obviously, if $\frac{\partial l_{1}(\mu, \varsigma)}{\partial \varsigma} \neq 0$, then the map $(\mu, \varsigma) \rightarrow\left(\frac{\xi(\mu)}{\omega(\mu)}\right.$, $\left.l_{1}(\mu, \varsigma)\right)$ at $(\mu, \varsigma)=(0,0)$ is

$$
\left(\begin{array}{cc}
\frac{\partial \frac{\xi(\mu)}{\omega(\mu)}}{\partial \mu} & \frac{\partial \frac{\xi(\mu)}{\omega(\mu)}}{\partial \varsigma} \\
\frac{\partial l_{1}(\mu, \varsigma)}{\partial \mu} & \frac{\partial l_{1}(\mu, \varsigma)}{\partial \varsigma}
\end{array}\right) \neq 0
$$

Theorem 3.2 If $l_{1}(0,0)=0, \frac{\partial l_{1}(\mu, \zeta)}{\partial \varsigma} \neq 0$ and $l_{2}(0,0) \neq 0$ are all satisfied, then system (1) undergoes Bautin bifurcation at the critical point $\left(\tau_{0}, r_{0}\right)$.

\section{Zero-Hopf bifurcation}

When the characteristic equation (6) has a simple zero root and a pair of pure imaginary roots and the other roots have negative real parts, the zero-Hopf bifurcation [25-27]will appear in system (1). With the emergence of zero-Hopf bifurcation, the dynamic phenomena of coexistence of periodic solutions and multistability are produced. Then, we study the existence of zero-Hopf bifurcation and calculate its normal form.

\subsection{Existence of zero-Hopf bifurcation}

To find the existence of zero-Hopf bifurcation, we need to study equation (6). Let

$$
G(\lambda, \tau)=\lambda^{2}-a_{22} \lambda+\left(d \lambda-a_{22} d\right) e^{-\lambda \tau}+a_{12} a_{21} .
$$

For (15), we have the following.

Theorem 4.1 When $d=\frac{a_{12} a_{21}}{a_{22}}$ and $a_{22}^{2}-a_{12} a_{21}<0$ hold, the function has a simple zero point for all $\tau \geq 0$.

Proof For (15), if $d=\frac{a_{12} a_{21}}{a_{22}}$, then we have

$$
G(0, \tau)=a_{12} a_{21}-a_{22} d=0
$$

and

$$
\frac{\partial G(0, \tau)}{\partial \lambda}=-a_{22}+\tau a_{12} a_{21}+d
$$

Due to $a_{22}^{2}-a_{12} a_{21}<0$, then $\left.\frac{\partial G(0, \tau)}{\partial \lambda}\right|_{d=\frac{a_{12} a_{21}}{a_{22}}}=0$ if and only if $\tau=\frac{a_{22}^{2}-a_{12} a_{21}}{a_{22}}<0$. So (15) has a simple zero point for all $\tau \geq 0$. Namely, $\lambda=0$ is a simple zero eigenvalue for all $\tau \geq 0$.

For $\tau=0$, if $d=\frac{a_{12} a_{21}}{a_{22}}$ and $a_{22}^{2}-a_{12} a_{21}<0$ are satisfied, the characteristic equation becomes

$$
\lambda\left(\lambda+d-a_{22}\right)=\lambda\left(\lambda+\frac{a_{12} a_{21}-a_{22}^{2}}{a_{22}^{2}}\right)=0 .
$$

Apparently, all roots of (16) are

$$
\lambda_{1}=0, \quad \lambda_{2}=\frac{a_{22}^{2}-a_{12} a_{21}}{a_{22}^{2}}<0 .
$$


Lemma 4.1 For $\tau=0$, if $d=\frac{a_{12} a_{21}}{a_{22}}$ and $a_{22}^{2}-a_{12} a_{21}<0$ hold, then (15) has a simple zero root and the other root with negative part.

Following Sect. 2, we have the following.

Lemma 4.2 For $\tau>0$, if $d=\frac{a_{12} a_{21}}{a_{22}}$ holds, then (15) has a simple zero root and a pair of purely imaginary roots $\pm i \omega_{0}$ at $\tau=\tau_{0}$, and the other roots of (15) have negative real parts. Therefore, system (1) undergoes a zero-Hopf bifurcation around the nontrivial equilibrium $E_{2}$ at $(\tau, r)=\left(\tau_{0}, r_{0}\right)$.

\subsection{Normal form of zero-Hopf bifurcation}

Still denote a zero-Hopf point by $\left(\tau_{0}, r_{0}\right)$, and use the previous symbol $\mu=\tau-\tau_{0}, \varsigma=r-r_{0}$. In this case, the eigenvalues of $A(0,0)$ are $i \omega_{0} \tau_{0}$ and 0 , and their corresponding eigenvectors are $\varphi_{1}(\theta)=(1, \rho)^{T} e^{i \omega \tau_{0} \theta}, \varphi_{2}(\theta)=(1, v)^{T}$. For the adjoint operator $A^{*}$, the eigenvalues of $A^{*}$ are $-i \omega_{0} \tau_{0}$ and 0 , and their corresponding eigenvectors are $\psi_{1}(\theta)=K_{1}(1, \sigma) e^{i \omega_{0} \tau_{0} s}$, $\psi_{2}(\theta)=K_{2}(1, c)$, where according to

$$
\begin{aligned}
& B_{1} \varphi_{1}(0)+B_{2} \varphi_{1}(-1)=i \omega_{0} \tau_{0} \varphi_{1}(0), \\
& \left(B_{1}+B_{2}\right)\left(\begin{array}{l}
1 \\
v
\end{array}\right)=0,
\end{aligned}
$$

and

$$
\begin{aligned}
& \psi_{1}(0) B_{1}+\psi_{1}(1) B_{2}=-i \omega_{0} \tau_{0} \psi_{1}(0), \\
& (1, c)\left(B_{1}+B_{2}\right)=0,
\end{aligned}
$$

we have

$$
\rho=\frac{d e^{-i \omega_{0} \tau_{0}}+i \omega_{0}}{a_{12}}, \quad v=\frac{d}{a_{12}}, \quad \sigma=\frac{d e^{i \omega_{0} \tau_{0}}-i \omega_{0}}{a_{12}}, \quad c=\frac{d}{a_{21}} .
$$

It is easy to verify $\left\langle\psi_{2}, \varphi_{1}\right\rangle=\left\langle\psi_{1}, \varphi_{2}\right\rangle=0$, and by

$$
\begin{aligned}
\left\langle\bar{\psi}_{1}, \varphi_{1}\right\rangle & =\bar{K}_{1}\left[(1, \bar{\sigma})\left(\begin{array}{c}
1 \\
\rho
\end{array}\right)+\int_{-1}^{0}(1, \bar{\sigma}) e^{-i \omega_{0} \tau_{0}(\zeta+1)} B_{2}\left(\begin{array}{l}
1 \\
\rho
\end{array}\right) e^{i \omega_{0} \tau_{0} \zeta} d \zeta\right] \\
& =\bar{K}_{1}\left[1+\bar{\sigma} \rho+d \tau_{0} e^{-i \omega_{0} \tau_{0}}\right]=1 \\
\left\langle\psi_{2}, \varphi_{2}\right\rangle & =K_{2}\left[\left(1, \frac{d}{a_{21}}\right)\left(\begin{array}{c}
1 \\
\frac{d}{a_{12}}
\end{array}\right)+\int_{-1}^{0}\left(1, \frac{d}{a_{21}}\right) B_{2}\left(\begin{array}{c}
1 \\
\frac{d}{a_{12}}
\end{array}\right) d \zeta\right] \\
& =K_{2}\left[1+\frac{d^{2}}{a_{21} a_{12}}+d \tau_{0}\right]=1,
\end{aligned}
$$

we have

$$
K_{1}=\frac{1}{1+\bar{\sigma} \rho+d \tau_{0} e^{-i \omega_{0} \tau_{0}}}, \quad K_{2}=\frac{a_{21} a_{12}}{\left(1+d \tau_{0}\right) a_{21} a_{12}+d^{2}} .
$$


Let $\Phi=\left(\varphi_{1}, \bar{\varphi}_{1}, \varphi_{2}\right), \Psi=\left(\bar{\psi}_{1}, \psi_{1}, \psi_{2}\right)$ with $\dot{\Phi}=\Phi J, \dot{\Psi}=-J \Psi,\langle\Psi, \Phi\rangle=1$, where $J=$ $\operatorname{diag}\left(i \omega_{0} \tau_{0},-i \omega_{0} \tau_{0}, 0\right)$. Furthermore, we set $\Lambda=\left\{0, i \omega_{0},-i \omega_{0}\right\}$, and $P$ is the generalized eigenspace associated with $\Lambda$ and $P^{*}$ is the space adjoint with $P$. Then, $C$ can be decomposed as $C=P \oplus Q$, where

$$
Q=\left\{\varphi \in C:\langle\psi, \varphi\rangle=0 \text {, for all } \psi \in P^{*}\right\} .
$$

Let $u=\Phi x+y$ with $x \in C^{3}, y \in Q$ and

$$
\Psi(0)=\left(\begin{array}{ll}
\bar{K}_{1} & \bar{K}_{1} \bar{\sigma} \\
K_{1} & K_{1} \sigma \\
K_{2} & v K_{2}
\end{array}\right)
$$

System (11) can be decomposed into

$$
\left\{\begin{array}{l}
\dot{x}=J x+\Psi(0) F(\Phi x+y, \mu) \\
\dot{y}=A_{Q} y+(1-\pi) X_{0} F(\Phi x+y, \mu)
\end{array}\right.
$$

namely

$$
\left\{\begin{array}{l}
\dot{x}=J x+\frac{1}{2} f_{2}^{1}(x, y, \mu, \varsigma)+\frac{1}{3} f_{2}^{2}(x, y, \mu, \varsigma)+\text { h.o.t, } \\
\dot{y}=A_{Q} y+\frac{1}{2} f_{2}^{2}(x, y, \mu, \varsigma)+\frac{1}{3} f_{3}^{2}(x, y, \mu, \varsigma)+\text { h.o.t }
\end{array}\right.
$$

where we focus on $f_{2}^{1}(x, y, \mu, \varsigma)$

$$
f_{2}^{1}(x, y, \mu, \varsigma)=\left(\begin{array}{l}
\bar{K}_{1} F_{2}^{1}(x, y, \mu, \varsigma)+\bar{K}_{1} \bar{\sigma} F_{2}^{2}(x, y, \mu, \varsigma) \\
K_{1} F_{2}^{1}(x, y, \mu, \varsigma)+K_{1} \bar{\sigma} F_{2}^{2}(x, y, \mu, \varsigma) \\
K_{2} F_{2}^{1}(x, y, \mu, \varsigma)+v K_{2} F_{2}^{2}(x, y, \mu, \varsigma)
\end{array}\right),
$$

and

$$
\begin{aligned}
\frac{1}{2} F_{2}^{1}= & \mu a_{12}\left(i \omega_{0} x_{1}-i \omega_{0} x_{2}+y_{2}(0)\right) \\
& -\mu d\left(e^{-i \omega_{0} \tau_{0}} x_{1}+e^{i \omega_{0} \tau_{0}} x_{2}+x_{3}+y_{1}(-1)\right) \\
& -a_{13}\left(\tau_{0}+\mu\right)\left(i \omega_{0} x_{1}-i \omega_{0} x_{2}+y_{2}(0)\right)^{2}, \\
\frac{1}{2} F_{2}^{2}= & \mu a_{21}\left(x_{1}+x_{2}+x_{3}+y_{1}(0)\right) \\
& +\mu\left(r_{0}+\varsigma\right) a_{22}^{*}\left(i \omega_{0} x_{1}-i \omega_{0} x_{2}+y_{2}(0)\right) \\
& -\beta\left(\tau_{0}+\mu\right)\left(x_{1}+x_{2}+x_{3}\right. \\
& \left.+y_{1}(0)\right)\left(i \omega_{0} x_{1}-i \omega_{0} x_{2}+y_{2}(0)\right) \\
& +\left(r_{0}+\varsigma\right)\left(\tau_{0}+\mu\right) a_{23}\left(i \omega_{0} x_{1}-i \omega_{0} x_{2}+y_{2}(0)\right)^{2} .
\end{aligned}
$$


Defining $M_{2}$ to be the operator in $V_{2}^{5}\left(\mathbb{R}^{3} \times \operatorname{Ker} \pi\right)$ with values in the same space by

$$
\left\{\begin{array}{l}
M_{2}(p, h)=\left(M_{2}^{1} p, M_{2}^{2} h\right) \\
M_{2}^{1}(p, \mu, \varsigma)=D_{x} p(x, \mu, \varsigma) J x-J p(x, \mu, \varsigma)
\end{array}\right.
$$

and on the center manifold, (18) can be transformed into the following normal form:

$$
\dot{x}=J x+\frac{1}{2} g_{2}^{1}(x, 0, \mu, \varsigma)+h . o . t,
$$

where

$$
\begin{aligned}
g_{2}^{1}(x, 0, \mu, \varsigma)= & \operatorname{Pr} o j_{\operatorname{Im}\left(M_{2}^{1}\right)} f_{2}^{1}(x, 0, \mu, \varsigma)+|(\mu, \varsigma)|^{2} \\
= & \left(\begin{array}{c}
\left(h_{10} \mu+h_{11} \varsigma\right) x_{1}+\left(h_{12}+h_{13}\right) x_{1}^{2} \\
+h_{15} x_{3}^{2}+h . o . t \\
\left(\bar{h}_{10} \mu+\bar{h}_{11} \varsigma\right) x_{2}+\left(\bar{h}_{12}+\bar{h}_{13}\right) x_{2}^{2} \\
-\left(2 \bar{h}_{12}+\bar{h}_{14}\right) x_{1} x_{2}+\text { h.o.t } \\
h_{21} \mu x_{3}+h_{22} x_{2} x_{3}
\end{array}\right)
\end{aligned}
$$

and

$$
\begin{aligned}
& h_{10}=\left(i a_{12} \omega_{0}-d e^{-i \omega_{0} \tau_{0}}+\bar{\sigma}\left(a_{21}+i \omega_{0} r_{0} a_{22}^{*}\right)\right) \bar{K}, \\
& h_{11}=\tau_{0} a_{22}^{*} \bar{\sigma} \bar{K}_{1}, \\
& h_{12}=\left(a_{13}-\bar{\sigma} r_{0} a_{23}\right) \tau_{0} \bar{K}_{1} \omega_{0}^{2}, \\
& h_{13}=i \beta \omega_{0} \bar{K}_{1} \bar{\sigma}, \\
& h_{14}=-\tau_{0} \omega_{0}^{2} a_{23} \bar{K}_{1} \bar{\sigma}, \\
& h_{15}=\left(r_{0} a_{23} \bar{\sigma}-a_{13}-\beta\right) \tau_{0} \bar{K}_{1}, \\
& h_{21}=0, \\
& h_{22}=i \beta \tau_{0} \omega_{0} v K_{2} .
\end{aligned}
$$

Equation (19) is rewritten as

$$
\left\{\begin{aligned}
\dot{x}_{1}= & i \omega_{0} \tau_{0} x_{1}+\left(h_{10} \mu+h_{11} \varsigma\right) x_{1}+\left(h_{12}+h_{13}\right) x_{1}^{2} \\
& +h_{15} x_{3}^{2}+\text { h.o.t } \\
\dot{x}_{2}= & -i \omega_{0} \tau_{0} x_{2}+\left(\bar{h}_{10} \mu+\bar{h}_{11} \varsigma\right) x_{2}+\left(\bar{h}_{12}+\bar{h}_{13}\right) x_{2}^{2} \\
& -\left(2 \bar{h}_{12}+\bar{h}_{14}\right) x_{1} x_{2}+\text { h.o.t } \\
\dot{x}_{3}= & h_{21} \mu x_{3}+h_{22} x_{2} x_{3}+\text { h.o.t. }
\end{aligned}\right.
$$

Carrying out the following coordinate transformation:

$$
\left\{\begin{array}{l}
x_{1}=r \cos \theta-i r \sin \theta, \\
x_{2}=r \cos \theta+i r \sin \theta, \\
x_{3}=w
\end{array}\right.
$$


and equation (20) becomes

$$
\left\{\begin{array}{l}
\dot{r}=g_{10} r+g_{20} r^{2}+g_{02} w^{2}+\text { h.o.t, } \\
\dot{w}=g_{01} w+g_{11} r w+\text { h.o.t, } \\
\dot{\theta}=-\omega \tau_{0}-\left[\operatorname{Im}\left\{\bar{h}_{10}\right\} \mu+\operatorname{Im}\left\{\bar{h}_{11}\right\} \varsigma\right]+\text { h.o.t, }
\end{array}\right.
$$

where

$$
\begin{aligned}
& g_{10}=\operatorname{Re}\left\{h_{10}\right\} \mu+\operatorname{Re}\left\{h_{11}\right\} \varsigma, \\
& g_{20}=3 h_{12}+h_{14}+\operatorname{Re}\left\{h_{13}\right\}, \\
& g_{01}=0, \quad g_{11}=\operatorname{Re}\left\{h_{22}\right\}, \quad g_{02}=h_{15} .
\end{aligned}
$$

Omitting the third equation of (20), we get the quadratic truncated normal form of zeroHopf bifurcation

$$
\left\{\begin{array}{l}
\dot{r}=g_{10} r+g_{11} r^{2}+\text { h.o.t } \\
\dot{w}=g_{20} w+g_{21} r w+\text { h.o.t }
\end{array}\right.
$$

Two quantities that distinguish the bifurcation are

$$
s=g_{20} g_{02}, \quad \theta=\frac{g_{11}}{g_{20}} .
$$

\section{Hopf-Hopf bifurcation}

From the point of view of bifurcation diagram, when two Hopf bifurcation curves intersect, a Hopf-Hopf bifurcation may occur at these intersections. Theoretically, in this situation, the characteristic equation (6) has two pairs of simple pure imaginary eigenvalues, denoted as $\pm i \omega_{1}$ and $\pm i \omega_{2}$, and the other eigenvalues have strictly negative real parts [2831]. In the following, we use the methods in [32] to analyze the Hopf-Hopf bifurcation.

Suppose that the eigenvectors corresponding to $A(0,0)$ and $A^{*}$ are $p_{1}, p_{2}, p_{1}^{*}, p_{2}^{*}$, respectively, and satisfy

$$
\begin{aligned}
& A(0,0) p_{1}=i \omega_{1} p_{1}, \quad A(0,0) p_{2}=i \omega_{2} p_{2}, \\
& A^{*} p_{1}^{*}=-i \omega_{1} p_{1}^{*}, \quad A^{*} p_{2}^{*}=-i \omega_{2} p_{2}^{*}
\end{aligned}
$$

then

$$
\begin{array}{ll}
p_{1}(\theta)=\left(1, \gamma_{1}\right)^{T} e^{i \omega_{1} \theta}, & p_{1}^{*}(s)=D_{1}\left(1, \gamma_{1}^{*}\right)^{T} e^{i \omega_{1} s}, \\
p_{2}(\theta)=\left(1, \gamma_{2}\right)^{T} e^{i \omega_{2} \theta}, & p_{2}^{*}(s)=D_{2}\left(1, \gamma_{2}^{*}\right)^{T} e^{i \omega_{2} s},
\end{array}
$$

where

$$
\begin{array}{ll}
\gamma_{1}=\frac{i \omega_{1}-d e^{i \omega_{1} \tau_{0}}}{a_{12}}, & \gamma_{1}^{*}=\frac{-i \omega_{1}-d e^{-i \omega_{1} \tau_{0}}}{a_{12}}, \\
\gamma_{2}=\frac{i \omega_{2}-d e^{i \omega_{2} \tau_{0}}}{a_{12}}, & \gamma_{2}^{*}=\frac{-i \omega_{2}-d e^{-i \omega_{2} \tau_{0}}}{a_{12}},
\end{array}
$$




$$
\bar{D}_{1}=\frac{1}{1+\bar{\gamma}_{1}^{*} \gamma_{1}-d \tau_{0} e^{-i \omega_{1} \tau_{0}}}, \quad \bar{D}_{2}=\frac{1}{1+\bar{\gamma}_{2}^{*} \gamma_{2}-d \tau_{0} e^{-i \omega_{2} \tau_{0}}},
$$

and

$$
\left\langle p_{1}^{*}, p_{1}\right\rangle=\left\langle p_{2}^{*}, p_{2}\right\rangle=1, \quad\left\langle p_{1}^{*}, p_{2}\right\rangle=\left\langle p_{2}^{*}, p_{1}\right\rangle=0
$$

Continuing to use the disturbance $\mu=\tau-\tau_{0}, \varsigma=r-r_{0}$, then, on the center manifold, we have

$$
\begin{aligned}
\dot{x}_{1}(t) & =\left\langle p_{1}^{*}, \dot{u}_{t}\right\rangle \\
& =\left\langle p_{1}^{*}, A u_{t}+R u_{t}\right\rangle \\
& =i \omega_{1} x_{1}(t)+\bar{p}_{1}^{*}(0) F\left(0, W^{(\mu, \varsigma)}(t, \theta)+2 \operatorname{Re}\left[x_{1}(t) p_{1}(0)+x_{2}(t) p_{2}(0)\right]\right) \\
& =i \omega_{1} x_{1}(t)+g^{1}\left(x_{1}(t), \bar{x}_{1}(t), \mu, \varsigma\right), \\
\dot{x}_{2}(t) & =\left\langle p_{2}^{*}, \dot{u}_{t}\right\rangle \\
& =\left\langle p_{2}^{*}, A u_{t}+R u_{t}\right\rangle \\
& =i \omega_{2} x_{1}(t)+\bar{p}_{2}^{*}(0) F\left(0, W^{(\mu, \varsigma)}(t, \theta)+2 \operatorname{Re}\left[x_{1}(t) p_{1}(0)+x_{2}(t) p_{2}(0)\right]\right) \\
& =i \omega_{2} x_{1}(t)+g^{2}\left(x_{2}(t), \bar{x}_{2}(t), \mu, \varsigma\right),
\end{aligned}
$$

where $g^{1}$ and $g^{2}$ are in the form of

$$
\begin{aligned}
& g^{j}\left(x_{j}(t), \bar{x}_{j}(t), \mu, \varsigma\right)=\sum_{l+s+r+k \geq 1} \frac{1}{l ! s ! r ! k !} g_{l s r k}^{j}(\mu, \varsigma) x_{1}^{l} \bar{x}_{1}^{s} x_{2}^{r} \bar{z}_{2}^{k}, \\
& j=1,2 .
\end{aligned}
$$

The third-order normal form near a Hopf-Hopf point is given by

$$
\left\{\begin{array}{l}
\dot{x}_{1}=i \omega_{1}+e_{2100} x_{1}\left|x_{1}\right|^{2}+e_{1011} x_{1}\left|x_{2}\right|^{2}+\text { h.o.t } \\
\dot{x}_{2}=i \omega_{2}+e_{1100} x_{2}\left|x_{1}\right|^{2}+e_{0021} x_{2}\left|x_{2}\right|^{2}+\text { h.o.t. }
\end{array}\right.
$$

Similarly, the key quantities to determine the bifurcation are

$$
\theta(0)=\frac{\operatorname{Re} e_{1011}}{\operatorname{Re} e_{0021}}, \quad \delta(0)=\frac{\operatorname{Re} e_{1110}}{\operatorname{Re} e_{2100}} .
$$

Remark 5.1 Here, we do not give the specific expressions of $e_{2100}, e_{1011}$ and $e_{1100}, e_{0021}$ in (21), and the calculation process can refer to the introduction of Hopf-Hopf bifurcation in reference [32].

\section{Simulations}

In this section, we perform some simulations to support the theoretical results about codimension two bifurcations in system (1). The simulations are divided into two parts, one for Bautin and zero-Hopf bifurcations, and the other for the Hopf-Hopf bifurcation. Moreover, the phenomena of multistability and periodic coexistence are also simulated. 


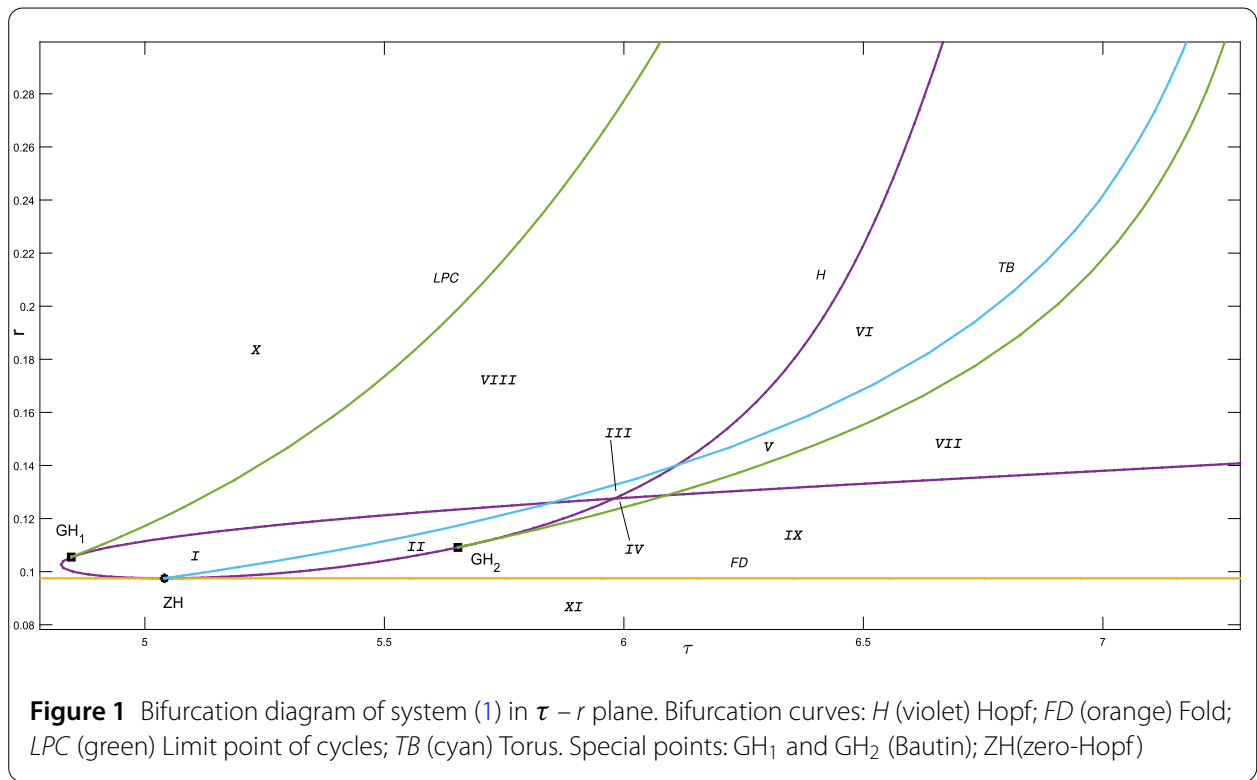

\subsection{Simulations of Bautin and zero-Hopf bifurcations}

We take $\tau$ and $r$ as bifurcation parameters to obtain the bifurcation diagram of system (1). Many papers [33, 34] have given the parameter values of this kind of tumor model. After analysis, the values of other parameters are as follows:

$$
\alpha=0.2499, \quad b=9.92, \quad d=0.2, \quad k=10, \quad m=0.45, \quad \beta=0.6 .
$$

By using DDE-BIFTOOL [35-37], the $\tau-r$ plane bifurcation diagram is shown in Fig. 1. From the diagram, we can see that there are two Bautin points and one zero-Hopf point of system (1), corresponding to $\mathrm{GH}_{1}, \mathrm{GH}_{2}$, and $\mathrm{ZH}$ respectively. In fact, for $\mathrm{GH}_{1}$, some calculations demonstrate $\tau=4.84628, r=0.10548$, and the first Lyapunov coefficient $\operatorname{Re} l_{1}(0,0) \approx 0$, the second Lyapunov coefficient $\operatorname{Re} l_{2}(0,0)=0.019362>0$. Similarly, for $\mathrm{GH}_{2}$, we have $\tau=5.65348, r=0.10914$ and $\operatorname{Re} l_{1}(0,0) \approx 0, \operatorname{Re} l_{2}(0,0)=-0.0091855>0$, and at the top right of the point, the Hopf bifurcation is supercritical, and the bottom left of this point, the Hopf bifurcation is subcritical. At $\mathrm{ZH}, \tau=5.0408, r=0.09749$, it is easy to verify that the content of Lemma 4.2 can be satisfied; moreover, we have $s=0.00062874$, $\theta=-0.6416$. Meanwhile the limit point of cycles, marked green, originating from Bautin bifurcation and the torus bifurcation, marked cyan, originating from the zero-Hopf bifurcation are also drawn.

Due to the appearance of these bifurcation curves, the $\tau-r$ plane is divided into 11 regions, as shown in Fig. 1, labeled I-XI. Next, we take different values of $(\tau, r)$ in these regions for numerical simulations. Using Matlab function DDE23, the simulation results are shown in Fig. 2, Fig. 3, and Fig. 4, and the black dot in the graph represents the initial value of the solution. Specifically, there are a stable periodic solution for the system in region I and coexistence of two unstable periodic solutions in region IX(see Fig. 2). Figure 3 shows the coexistence of two unstable periodic solutions in region II, one of which is eventually stable to the origin, which reveals that immune cells and tumor cells go to apoptosis together, an unstable periodic solution and an asymptotically stable solution in region III, and shows two opposite phenomena, one of which is asymptotically stable, and the other 


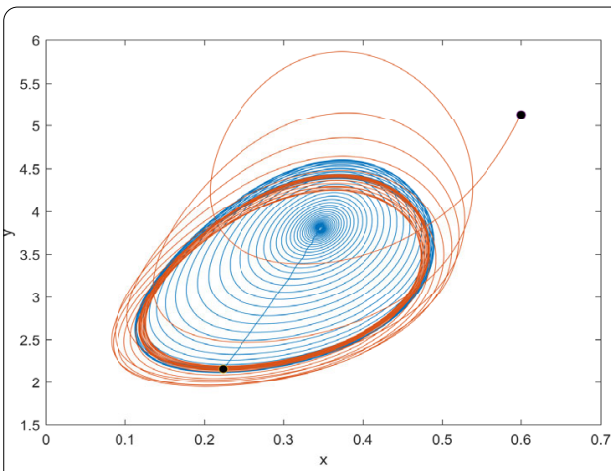

(a) $I-1$

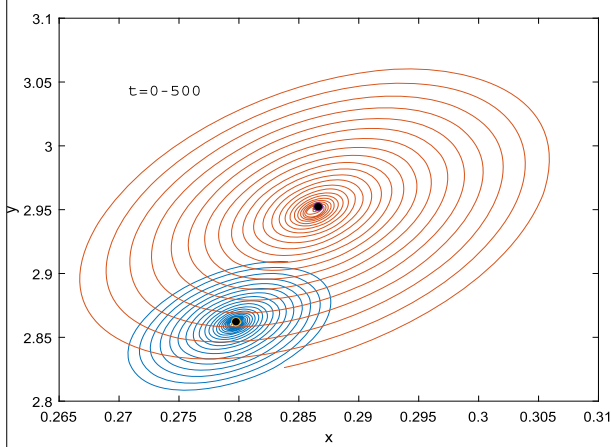

(c) $I X-1$

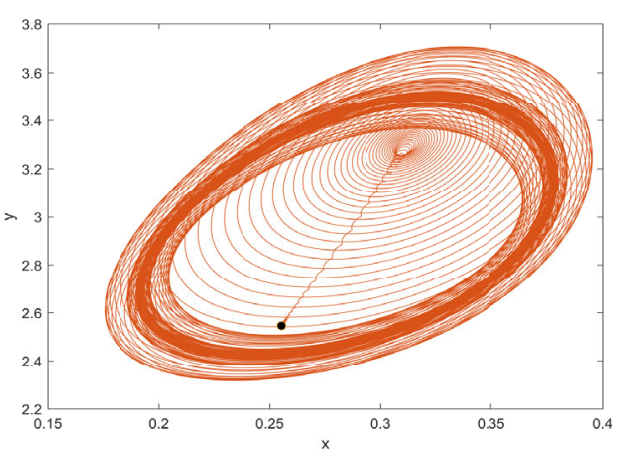

(b) $I-2$

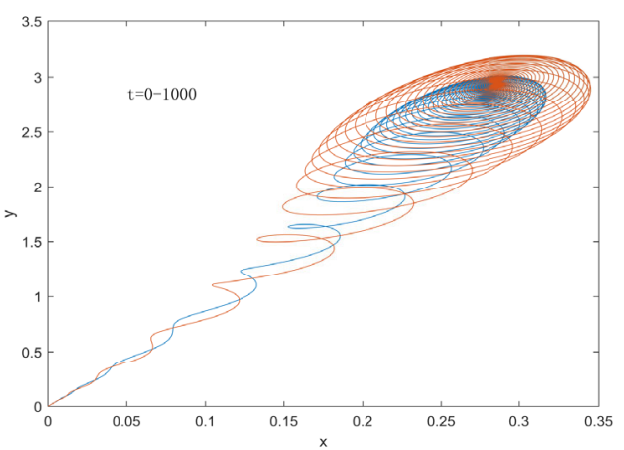

(d) $I X-2$

Figure 2 Trajectories of system (1) with different parameters and initializations in regions ( $/$ and $I X$ ) of Fig. 1, where the $(\tau, r)$ of $I, I X$ are $(5,0.1)$ and $(5.15,0.0975)$, respectively. (a)The initial values of the variable are $(0.6001,5.1389)$ and $(0.2333,2.159)$, and they finally stabilize to periodic solutions with large amplitude and small amplitude respectively. (b)The initial value of the variable is $(0.25526,2.5469)$. In fact, it is similar to (a), but the periodic solution fluctuates many times before it is stable. (c) and (d) have the same initial values $(0.2797,2.862)$ and $(0.286326,3.04507)$. (c) Coexistence of two unstable solutions. (d)With the increase of time, it finally stabilizes to the origin

is unstable, corresponding to regions IV and $\mathrm{X}$, respectively. An asymptotically stable solution and an unstable periodic solution are obtained in region $\mathrm{V}$, an asymptotically stable solution appearing in region VII, and an unstable periodic solution and an asymptotically stable solution showing in region VII, two stable periodic solutions coexisting in region VIII(see Fig. 4). Finally, for region XI, after calculation, we find that there is no positive equilibrium in this region, so the simulation is not carried out.

In a conclusion, in general, the larger the values of $\tau$ and $r$, that is, the closer the values of $(\tau, r)$ in Fig. 1 to the upper right regions, the more likely the solutions to be unstable, such as regions III, IV and V, VII(Although there may be stable solutions in a small range of positive equilibrium). On the contrary, when $\tau$ and $r$ are small, the solutions are asymptotically stable or periodically stable, such as regions I, II, and VIII. Therefore, if the tumor cell proliferation rate and the delay of immune cell apoptosis are small, it is conducive to the tumor in a stable state.

\subsection{Simulation of Hopf-Hopf bifurcation}

For Hopf-Hopf bifurcation, we simply give a numerical example to illustrate the existence of Hopf-Hopf bifurcation and draw a bifurcation diagram near the bifurcation point, as 


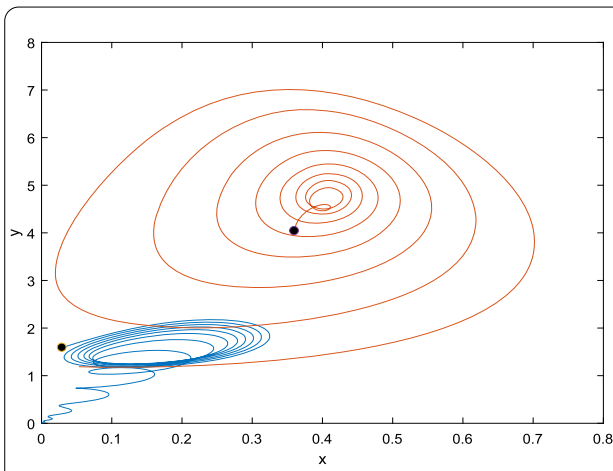

(a) $I I:(\tau, r)=(5.52,0.108)$

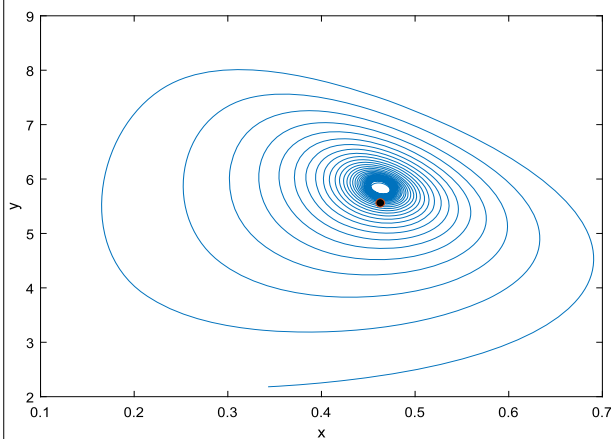

(c) $I V:(\tau, r)=(5.98,0.124)$

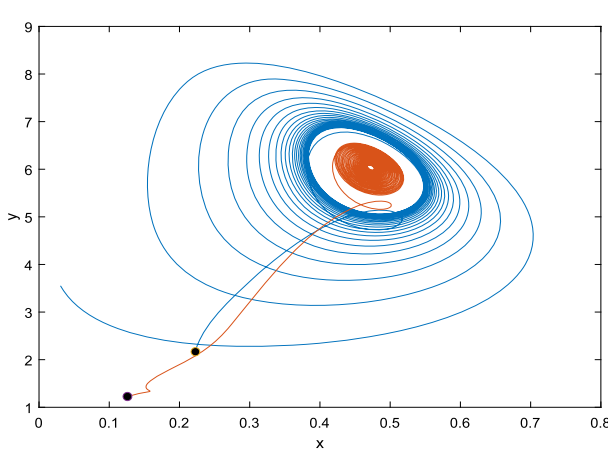

(b) $I I I:(\tau, r)=(5.94,0.128)$

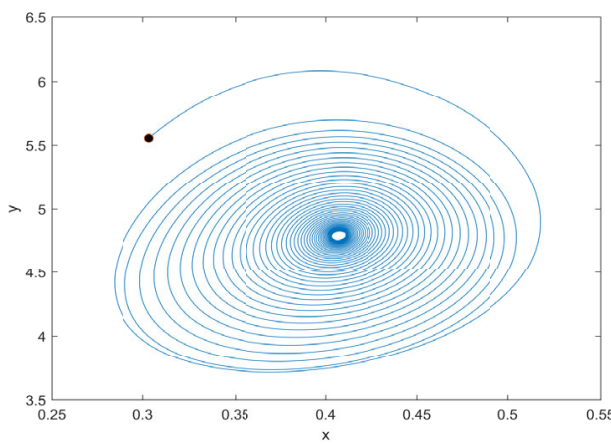

(d) $X:(\tau, r)=(4.8,0.108)$

Figure 3 Trajectories of system (1) with different parameters and initializations in regions (II-IV and X) of Fig. 1. For convenience, parameter values are given directly at the label of the graph. (a)Two unstable periodic solutions coexist with the initial value $(0.2932,1.5887)$ and $(0.3601,4.038)$, respectively, one of which is stable to the origin. (b)An unstable periodic solution with the initial value $(0.2233,2.1588$ ) and an asymptotically stable solution with the initial value $(0.1265,1.2177)$. (c) and (d) have opposite phenomena, one which is asymptotically stable with the initial value $(0.3034,5.555)$, and the other is unstable with the initial value $(0.4632,5.5445)$

shown in Fig. 5. The two Hopf bifurcation curves intersect, and the Hopf-Hopf bifurcation is generated at the intersection. Meanwhile, the two torus bifurcation curves originating from the bifurcation point are also given in Fig. 5. The parameter values of this example are as follows: $\alpha=0.7599, b=6.92, d=0.85, k=10, m=0.15, \beta=0.45$. At the HopfHopf point, we can calculate $\tau=10.734, r=0.055996$. Furthermore, two quantities that characterize the bifurcation are $\theta(0)=4.6571, \delta(0)=0.061712$. Following [32], the periodic phenomena in different regions $(D 1-D 6)$ near the Hopf-Hopf point can be observed.

In fact, system (1) has more than one Hopf-Hopf bifurcation. With the increase of time delay, more Hopf-Hopf points appear in the two-parameter plane $\tau-r$, so more complex phenomena of periodic solutions and chaos will exist in the system.

\section{Conclusion and discussion}

Co-dimension two bifurcations are a common bifurcation phenomenon in delay differential equations, which leads to complex dynamic behavior of the system. In this paper, a delayed tumor model is established, and the Allee effect is considered in this model. It is found that there are at most two positive equilibria in the system, and the stability condition of the equilibria is given. Due to the time delay and Allee effect, the system 


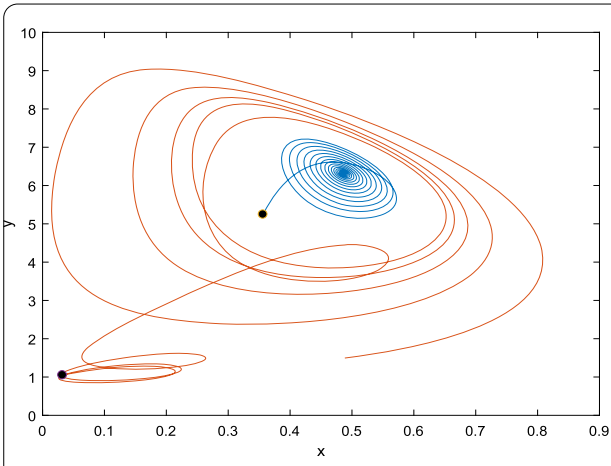

(a) $V:(\tau, r)=(6.1,0.135)$

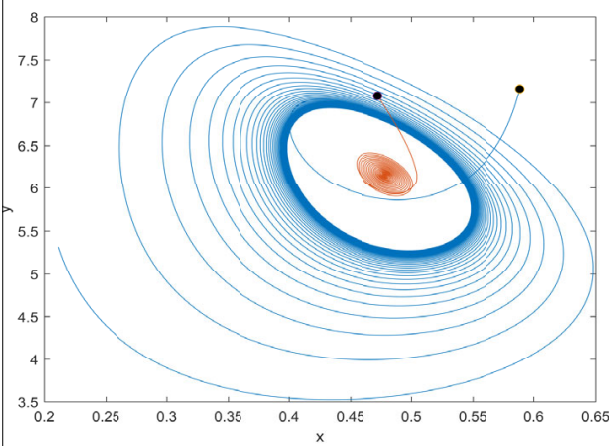

(c) VII: $(\tau, r)=(6.2,0.131)$

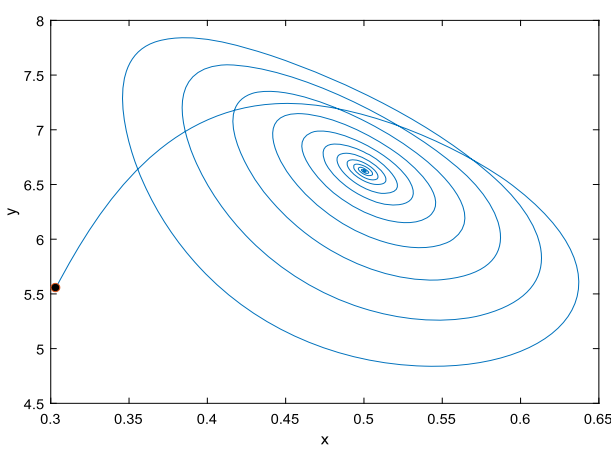

(b) $V I:(\tau, r)=(6.16,0.144)$

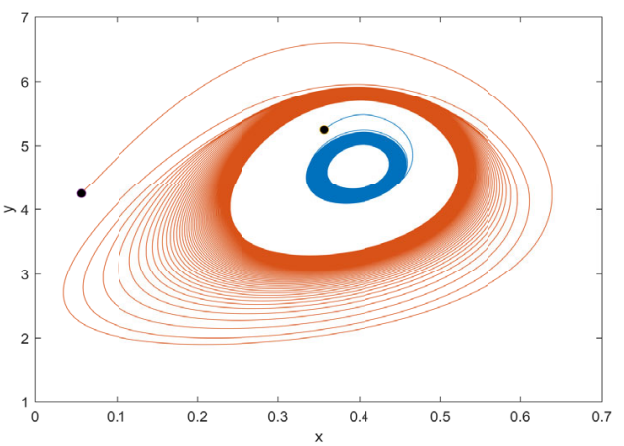

(d) VIII: $(\tau, r)=(4.866,0.1067)$

Figure 4 Trajectories of system (1) with different parameters and initializations in regions (V-VIII) of Fig. 1. (a)An asymptotically stable solution with the initial value $(0.256326,2.74507)$ and an unstable periodic solution with the initial value $(0.032247,1.0489)$. (b)An asymptotically stable solution with the initial value $(0.30326,5.5545)$. (c)Similarly to (a), an asymptotically stable solution with the initial value $(0.472656,7.07979)$ and an unstable periodic solution with the initial value $(0.58869,7.1607)$. (d)Two stable periodic solutions coexist with the initial values $(0.306325,5.545)$ and $(0.380326,4.505)$, respectively

has abundant co-dimension two bifurcations, accompanied by multistability and periodic coexistence. Particularly, after adding Allee effect, the system changes from a single equilibrium to two different positive equilibria, which is an important reason for the appearance of coexistence, and the time-delay also makes the original two-dimensional ordinary differential equation more complex, such as the emergence of zero-Hopf bifurcation and Hopf-Hopf bifurcation. We calculate the normal form of Bautin bifurcation, zero-Hopf bifurcation, and Hopf-Hopf bifurcation of the system. For Bautin bifurcation and zeroHopf bifurcation, the two-parameter bifurcation diagram is also given in numerical experiments in Sect. 6. By the simulation of different regions, the multistability and periodic coexistence are demonstrated. For Hopf-Hopf bifurcation, we only perform a numerical example. In fact, there are stable periodic solutions and chaos near the Hopf-Hopf bifurcation point.

To conclude, this paper analyzes the stability and bifurcations of a tumor model. The theoretical and simulation results are of biological significance, which is helpful for the future tumor research at the theoretical level. More specifically, the time-delay and growth rate of tumor cells play a key role in the development of tumor, which is conducive to our further research in the future. 


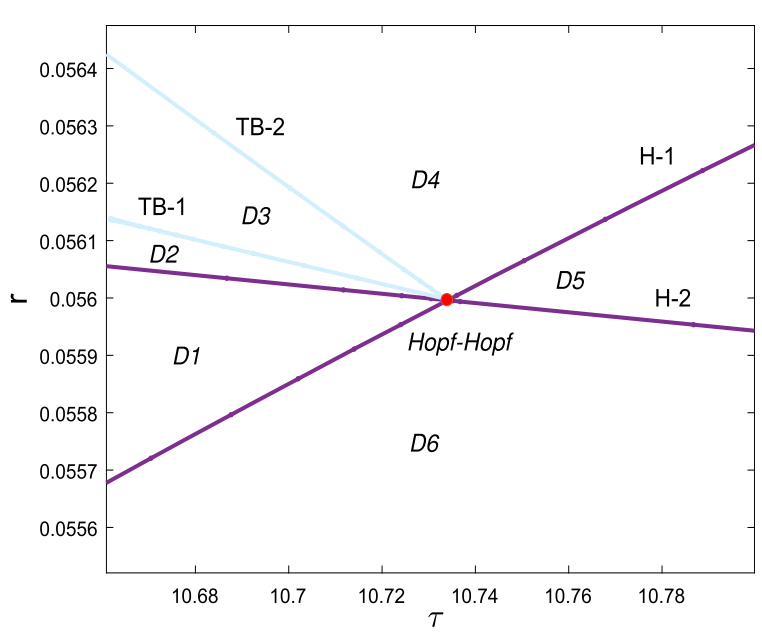

Figure 5 Bifurcation diagram of the Hopf-Hopf point. $\mathrm{H}-1$ and $\mathrm{H}-2$ represent two Hopf bifurcation curves, and their intersection is the Hopf-Hopf bifurcation. There are two torus bifurcation curves originating from the Hopf-Hopf point, TB-1 and TB-2. $\tau-r$ plane is divided into six regions, defined as D1 - D6

\section{Acknowledgements}

The authors are grateful to all anonymous reviewers for their valuable comments which have provided great help for the improvement of the paper.

\section{Funding}

This work is supported by Excellent Young and Middle-aged Scientific and Technological Innovation Team of Colleges and Universities of Hubei Province(T2020046)-Artificial Intelligence and Robot.

\section{Availability of data and materials}

All data generated or analysed during this study are included in this published article.

\section{Declarations}

\section{Competing interests}

The authors declare that they have no conflict of interest. The authors certify that this manuscript is original and has not been published and will not be submitted elsewhere for publication while being considered by Advances in Difference Equations, and all data supporting the findings of this study are included in this manuscript.

\section{Authors' contributions}

All authors read and approved the final manuscript.

\section{Publisher's Note}

Springer Nature remains neutral with regard to jurisdictional claims in published maps and institutional affiliations.

Received: 9 August 2021 Accepted: 4 November 2021 Published online: 07 December 2021

\section{References}

1. Siegel, R.L., Miller, K.D., Jemal, A.: Cancer statistics, 2019. CA Cancer J. Clin. 69(1), 7-34 (2019)

2. Chalmers, Z.R., Connelly, C.F., Fabrizio, D., Gay, L., Ali, S.M., Ennis, R., Schrock, A., Campbell, B., Shlien, A., Chmielecki, J., et al.: Analysis of 100,000 human cancer genomes reveals the landscape of tumor mutational burden. Gen. Med. 9(1), $1-14(2017)$

3. Dunn, G.P., Bruce, A.T., Ikeda, H., Old, L.J., Schreiber, R.D.: Cancer immunoediting: from immunosurveillance to tumor escape. Nat. Immunol. 3(11), 991-998 (2002)

4. Pietras, K., Östman, A.: Hallmarks of cancer: interactions with the tumor stroma. Exp. Cell Res. 316(8), 1324-1331 (2010)

5. d'Onofrio, A.: A general framework for modeling tumor-immune system competition and immunotherapy: mathematical analysis and biomedical inferences. Physica D 208(3-4), 220-235 (2005)

6. Makhlouf, A.M., El-Shennawy, L., Elkaranshawy, H.A.: Mathematical modelling for the role of CD4+ t cells in tumor-immune interactions. Comput. Math. Methods Med. 2020, Article ID 7187602 (2020)

7. Ashyani, A., RabieiMotlagh, O., Mohammadinejad, H.: A mathematical approach to effects of CTLs on cancer virotherapy in the second injection of virus. J. Theor. Biol. 453, 78-87 (2018)

8. Sardar, M., Biswas, S., Khajanchi, S.: The impact of distributed time delay in a tumor-immune interaction system. Chaos Solitons Fractals 142, 110483 (2021) 
9. Khajanchi, S., Nieto, J.J.: Mathematical modeling of tumor-immune competitive system, considering the role of time delay. Appl. Math. Comput. 340, 180-205 (2019)

10. Zhao, X.E., Hu, B.: Symmetry-breaking bifurcation for a free-boundary tumor model with time delay. J. Differ. Equ. 269(3), 1829-1862 (2020)

11. Kemwoue, F.F., Dongo, J.M., Mballa, R.N., Gninzanlong, C.L., Kemayou, M.W., Mokhtari, B., Biya-Motto, F., Atangana, J.: Bifurcation, multistability in the dynamics of tumor growth and electronic simulations by the use of PSpice. Chaos Solitons Fractals 134, 109689 (2020)

12. Zeng, C., Ma, S.: Dynamic analysis of a tumor-immune system under Allee effect. Math. Probl. Eng. 2020, Article ID 4892938 (2020)

13. Johnson, K.E., Howard, G., Mo, W., Strasser, M.K., Lima, E.A., Huang, S., Brock, A.: Cancer cell population growth kinetics at low densities deviate from the exponential growth model and suggest an Allee effect. PLoS Biol. 17(8), 3000399 (2019)

14. Böttger, K., Hatzikirou, H., Voss-Böhme, A., Cavalcanti-Adam, E.A., Herrero, M.A., Deutsch, A.: An emerging Allee effect is critical for tumor initiation and persistence. PLoS Comput. Biol. 11(9), 1004366 (2015)

15. Bozkurt, F., Yousef, A.: Flip bifurcation and stability analysis of a fractional-order population dynamics with Allee effect. J. Interdiscip. Math. 22(6), 1009-1029 (2019)

16. Hilker, F.M., Langlais, M., Malchow, H.: The Allee effect and infectious diseases: extinction, multistability, and the (dis-) appearance of oscillations. Am. Nat. 173(1), 72-88 (2009)

17. Sardar, M., Khajanchi, S.: Is the Allee effect relevant to stochastic cancer model? J. Appl. Math. Comput., 1-23 (2021)

18. Tsur, N., Kogan, Y., Rehm, M., Agur, Z.: Response of patients with melanoma to immune checkpoint blockade-insights gleaned from analysis of a new mathematical mechanistic model. J. Theor. Biol. 485, 110033 (2020)

19. Khajanchi, S., Banerjee, S.: Stability and bifurcation analysis of delay induced tumor immune interaction model. Appl. Math. Comput. 248, 652-671 (2014)

20. Khajanchi, S.: Modeling the dynamics of glioma-immune surveillance. Chaos Solitons Fractals 114, 108-118 (2018)

21. De Pillis, L.G., Radunskaya, A.: The dynamics of an optimally controlled tumor model: a case study. Math. Comput. Model. 37(11), 1221-1244 (2003)

22. Yang, X., Yang, M., Liu, H., Liao, X.: Bautin bifurcation in a class of two-neuron networks with resonant bilinear terms. Chaos Solitons Fractals 38(2), 575-589 (2008)

23. Song, Z., Xu, J.: Bursting near Bautin bifurcation in a neural network with delay coupling. Int. J. Neural Syst. 19(5), 359-373 (2009)

24. Zhen, B., Xu, J.: Bautin bifurcation analysis for synchronous solution of a coupled FHN neural system with delay. Commun. Nonlinear Sci. Numer. Simul. 15(2), 442-458 (2010)

25. Wu, X., Wang, L.: Zero-Hopf bifurcation for van der Pol's oscillator with delayed feedback. J. Comput. Appl. Math. 235(8), 2586-2602 (2011)

26. Zhen, B., Xu, J.: Fold-Hopf bifurcation analysis for a coupled Fitzhugh-Nagumo neural system with time delay. Int. J. Bifurc. Chaos 20(12), 3919-3934 (2010)

27. Bramburger, J., Dionne, B., LeBlanc, V.G.: Zero-Hopf bifurcation in the van der Pol oscillator with delayed position and velocity feedback. Nonlinear Dyn. 78(4), 2959-2973 (2014)

28. Zhang, L., Zhang, Z., Huang, L.: Double Hopf bifurcation of time-delayed feedback control for Maglev system. Nonlinear Dyn. 69(3), 961-967 (2012)

29. Xu, J., Chung, K.-W., Chan, C.-L.: An efficient method for studying weak resonant double Hopf bifurcation in nonlinear systems with delayed feedbacks. SIAM J. Appl. Dyn. Syst. 6(1), 29-60 (2007)

30. Chen, M., Wu, R., Liu, B., Chen, L.: Hopf-Hopf bifurcation in the delayed nutrient-microorganism model. Appl. Math. Model. 86, 460-483 (2020)

31. Guo, S., Chen, Y., Wu, J.: Two-parameter bifurcations in a network of two neurons with multiple delays. J. Differ. Equ. 244(2), 444-486 (2008)

32. Bosschaert, M., Wage, B., Kuznetsov, Y.: Description of the extension ddebiftool_nmfm (2015)

33. Banerjee, S., Khajanchi, S., Chaudhuri, S.: A mathematical model to elucidate brain tumor abrogation by immunotherapy with T11 target structure. PLoS ONE 10(5), 0123611 (2015)

34. Yu, M., Dong, Y., Takeuchi, Y.: Dual role of delay effects in a tumour-immune system. J. Biol. Dyn. 11(suppl. 2), 334-347 (2017)

35. Engelborghs, K., Luzyanina, T., Roose, D.: Numerical bifurcation analysis of delay differential equations using DDE-BIFTOOL. ACM Trans. Math. Softw. 28(1), 1-21 (2002)

36. Engelborghs, K., Luzyanina, T., Samaey, G., et al.: DDE-BIFTOOL: a Matlab package for bifurcation analysis of delay differential equations. TW Rep. 305, 1-36 (2000)

37. Wage, B.: Normal form computations for delay differential equations in DDE-BIFTOOL. Master's thesis (2014) 